\title{
The cost effectiveness of opportunistic chlamydia screening in England
}

\author{
Elisabeth J Adams, Katherine M E Turner, W John Edmunds
}

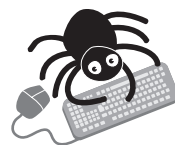

An appendix can be found on our website http://sti.bmj.com.

See end of article for authors' affiliations

Correspondence to:

W J Edmunds, Modelling \&

Economics Unit, Health

Protection Agency, 61

Colindale Avenue,

Colindale, London NW9

5EQ, UK; john.edmunds@

hpa.org.uk

Accepted 27 March 2007

Published Online First

2 April 2007

........................

Sex Transm Infect 2007;83:267-275. doi: 10.1136/sti.2006.024364

Background/aim: The National Chlamydia Screening Programme (NCSP) is being implemented in England. This study aims to estimate the cost effectiveness of (a) the NCSP strategy (annual screening offer to men and women aged under 25 years) and (b) alternative screening strategies.

Methods: A stochastic, individual based, dynamic sexual network model was combined with a cost effectiveness model to estimate the complications and associated costs of chlamydial infection. The model was constructed and parameterised from the perspective of the National Health Service (NHS) (England), including the direct costs of infection, complications and screening. Unit costs were derived from standard data sources and published studies. The average and incremental cost effectiveness ratio (cost per major outcome averted or quality adjusted life year (QALY) gained) of chlamydia screening strategies targeting women and/or men of different age groups was estimated. Sensitivity analyses were done to explore model uncertainty.

Results: All screening strategies modelled are likely to cost the NHS money and improve health. If pelvic inflammatory disease (PID) progression is less than $10 \%$ then screening at any level is unlikely to be cost effective. However, if PID progression is 10\% or higher the NCSP strategy compared to no screening appears to be cost effective. The incremental cost effectiveness analysis suggests that screening men and women aged under 20 years is the most beneficial strategy that falls below accepted thresholds. There is a high degree of uncertainty in the findings.

Conclusions: Offering an annual screening test to men and women aged under 20 years may be the most cost effective strategy (that is, under accepted thresholds) if PID progression is $10 \%$ or higher.
$\mathrm{T}$ he prevalence of genital chlamydial infection is $3-10 \%$ in women under 25 years old in England. ${ }^{1}$ Since many cases are asymptomatic, chlamydia screening is a way of identifying undiagnosed infection so that individuals and their partners can be treated. Earlier treatment prevents complications and reduces onward transmission. The National Chlamydia Screening Programme (NCSP) is currently being implemented in England. ${ }^{2}{ }^{3}$ However, questions remain regarding its cost effectiveness and that of alternative screening strategies. A transmission dynamic model of chlamydial infection in a sexually active population was used previously to estimate the impact of different screening strategies on chlamydia prevalence. ${ }^{45}$ These results were used in this analysis to estimate the complications from untreated chlamydial infection, and the costs associated with acute infection, clinical complications, and screening activities. A cost effectiveness analysis was performed to compare different screening strategies, in the context of limited resources.

\section{METHODS}

\section{Transmission dynamic model}

A stochastic, individual based, dynamic sexual network model was developed to simulate sexual behaviour and chlamydia transmission in England. The full methodology is explained elsewhere, including details of the extensive fitting process used to model sexual behaviour and chlamydia epidemiology realistically. ${ }^{4}$ The model simulated a heterosexual population of 20000 men and 20000 women aged 16-44. The rate of sexual partner change was highest in the youngest cohorts and decreased with age. Infection in the model was transmitted within discordant partnerships, assuming no acquired immunity to chlamydia. Symptomatic infection was assumed to have a shorter average duration than asymptomatic infection
( 1 month vs 6 months), because of active treatment seeking. Without screening effective partner notification (notification plus treatment of infected partners) was assumed to be $20 \%$. The overall chlamydia prevalence in the model was 3.2\% among all individuals, highest in 16-19 year olds and decreasing with age. $^{4}$

Three opportunistic screening strategies were modelled, targeting different age groups $(<20,<25,<30,<35,<40$ years old):

- Strategy 1 Offer an annual screen to women

- Strategy 2 Offer an annual screen to women and if they have changed their partner in the last 6 months

- Strategy 3 Offer an annual screen to women and men.

It was assumed that $85 \%$ of the population attended a healthcare site annually. ${ }^{5}$ Of those eligible for screening, a proportion ( $50 \%$ at baseline) accepted the screen. Thus, under strategy 1 the minimum interval between screens was 1 year. Once eligible, individuals attend approximately twice a year, but accept $50 \%$ of the time, hence the average time between screens was 2 years. Each subsequent screening offer was assumed to be independent of previous offers or acceptances.

Evidence from a recent study indicated that women have a greater risk of infection and reinfection if they have acquired a new partner. ${ }^{6}$ Strategy 2 extends screening eligibility based on sexual behaviour, to target those at highest risk. The NCSP

Abbreviations: CER, cost effectiveness ratio; EP, ectopic pregnancy; ICER, incremental cost effectiveness ratio; MO, major outcome; MOA, major outcomes averted; NCSP, National Chlamydia Screening Programme; PID, pelvic inflammatory disease; PN, partner notification; QALY, quality adjusted life year; TFI, tubal factor infertility 
A

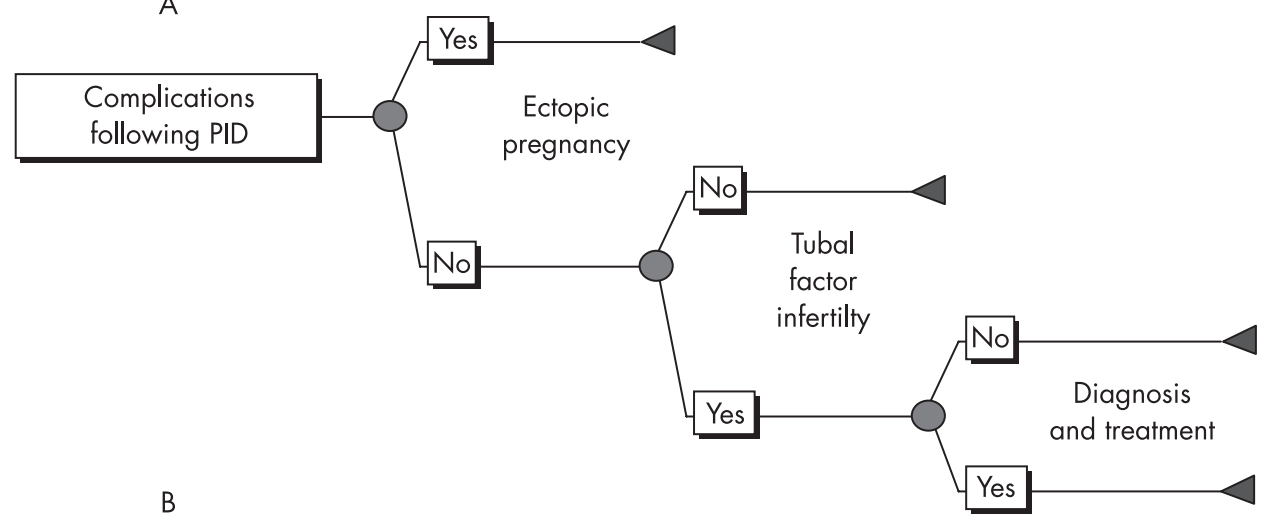

Figure 1 Flow of complications in (A) women with pelvic inflammatory disease (PID), and (B) neonates exposed to infected mothers.

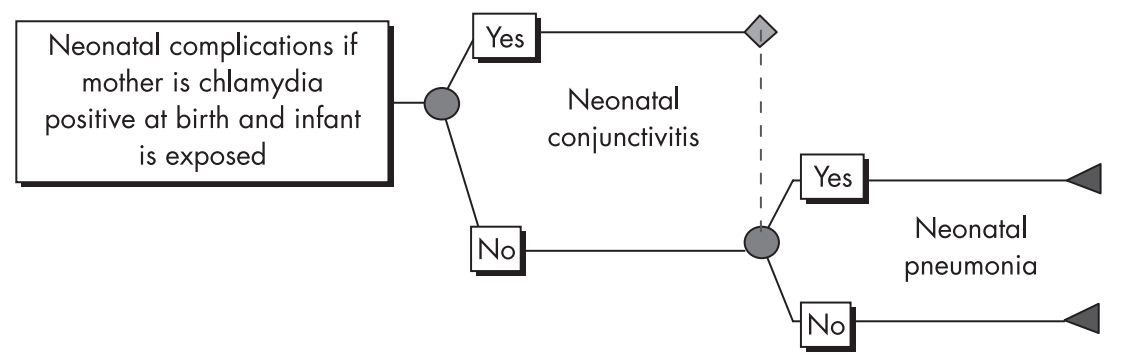

recommendation of an annual screen for men and women under 25 years old ${ }^{2}$ (strategy 3) was chosen as the baseline screening strategy for sensitivity analyses. Results of the sensitivity analyses performed on screening effectiveness have been reported previously. ${ }^{5}$ The probability of accepting a screen when offered was changed for both men and women from $50 \%$ (baseline) to $10 \%, 30 \%$, and $70 \%$. An additional, pessimistic scenario of $10 \%$ of women and $1.4 \%$ of men accepting was also modelled, which roughly approximates the number of screens performed in men and women in the NCSP in 2004-5. ${ }^{7}$ The efficacy of partner notification (PN) and treatment with screening introduction was changed from $20 \%$ to $50 \%$ (applied to partners of those screened and those actively seeking treatment). A final scenario examined the cost effectiveness when individuals only accepted a screen once, since evidence suggested that acceptance declines after the first screen acceptance. $^{8}$

\section{Cost effectiveness model}

A cost effectiveness model was constructed in Excel to estimate the costs of acute infection, the number of complications and their associated costs, and the costs of interventions under different screening strategies compared to no screening. The number of female and neonatal complications was modelled using a decision tree (Precision Tree, Palisade software) (fig l).

Only symptomatic pelvic inflammatory disease (PID) was modelled, as there is evidence from Westrom et al that the severity of PID symptoms is directly related to the probability of further complications such as ectopic pregnancy (EP) and tubal factor infertility (TFI). ${ }^{9}$ Furthermore, the causal link between undetected asymptomatic PID and TFI is weak. There is conflicting evidence about the proportion of chlamydia cases that result in PID. ${ }^{10-12}$ Therefore, three scenarios were run for no screening and each screening strategy with a PID progression probability of $1 \%, 10 \%$, and $30 \%$. To determine which assumption may be closest to the actual value, the number of cases of PID estimated by the model (no screening) was compared to an estimate of the annual incidence of PID in 1644 year olds of between 1500 and 2400 per 100000 women, from a GP based study. ${ }^{13}$ This included all clinical diagnoses of PID from any cause, and also those who might have been misdiagnosed (none of these cases were confirmed laproscopically).

The dynamic model output the incident cases of symptomatic and asymptomatic chlamydial infection in men and women, and acute complications (symptomatic PID in women and epididymitis in men), by year for each simulation. These cases in women were then used to estimate the number of cases of EP, TFI, neonatal conjunctivitis, and neonatal pneumonia. The probabilities of complications are given in table 1 and supporting evidence in the appendix (available at http:// sti.bmj.com/supplemental). Because of the stochastic nature of infection within the dynamic model, each simulation of the dynamic model resulted in a different number of cases of

Table 1 Risk of developing complications following acute chlamydial infection

\begin{tabular}{|c|c|c|c|c|}
\hline Complication & $\begin{array}{l}\text { Probability } \\
\text { (sample size) }\end{array}$ & Probability applied to: & Distribution type & Reference \\
\hline $\begin{array}{l}\text { Symptomatic PID (women) } \\
\text { Ectopic pregnancy (women) } \\
\text { Tubal factor infertility (women) } \\
\text { Neonatal conjunctivitis } \\
\text { Neonatal pneumonia } \\
\text { Epididymitis (men) }\end{array}$ & $\begin{array}{l}1 \%, 10 \%, 30 \% \\
7.6 \%(1309) \dagger \\
10.8 \%(1309) \dagger \\
14.8 \%(1055) \ddagger \\
7.0 \%(597) \ddagger \\
2 \%\end{array}$ & $\begin{array}{l}\text { Asymptomatic chlamydial infection } \\
\text { Symptomatic PID } \\
\text { Symptomatic PID (exclude those with EP) } \\
\text { Infected women giving birth vaginally } \\
\text { Infected women giving birth vaginally } \\
\text { Asymptomatic chlamydial infection }\end{array}$ & $\begin{array}{l}\text { Scenario analysis* } \\
\text { Beta } \\
\text { Beta } \\
\text { Beta } \\
\text { Beta } \\
\text { Fixed }\end{array}$ & $\begin{array}{l}\text { Assumption } \\
\text { Weström et ap } \\
\text { Weström et } a^{p} \\
\text { Rosenman et } a^{88} \\
\text { Rosenman et } a^{8} \\
\text { Assumption based on } \\
\text { work by Welte et } a^{1920}\end{array}$ \\
\hline
\end{tabular}

$P I D$, pelvic inflammatory disease, EP, ectopic pregnancy. *All screening strategies were run with all three probabilities. $†$ Based on the number of women trying to conceive, after a laparoscopically diagnosed PID case, the total denotes the total number followed up. $\ddagger$ The total is the number of infants exposed at birth. 
Table 2 Estimated average costs of acute infection, complications and interventions

\begin{tabular}{lc}
\hline Condition & Baseline cost (£) (SD) \\
\hline Acute conditions & \\
Symptomatically infected and actively seeking & \\
treatment for chlamydial infection & \\
Men & $64(6)$ \\
Women & $61(5)$ \\
Screened and infected (men/women) & $31(2)$ \\
Screened and not infected (men/women) & $20(2)$ \\
Do not accept screen offer (men/women) & $6(1)$ \\
Partner treatment & $27(2)$ \\
Complications & $137(46)$ \\
Pelvic inflammatory disease & $142(67)$ \\
Epididymitis & $762(329)$ \\
Ectopic pregnancy & $10798(4279)$ \\
Tubal factor infertility & $41(4)$ \\
Neonatal conjunctivitis & $612(555)$ \\
Neonatal pneumonia &
\end{tabular}

infection. The dynamic model was run 100 times for each scenario, and the average of these was input into the model to get base case results.

The model was constructed and parameterised from the perspective of the National Health Service in England, and included the direct costs of infection, complications, and screening. Unit costs were derived from standard data sources $^{14-16}$ and other published studies. Costs to the patient and wider society were not included in this analysis as recommended by the National Institute for Health and Clinical Excellence (NICE). ${ }^{17}$ Estimates of the average costs of acute conditions, complications, and interventions are given in table 2 and further details including how they were derived are given in the appendix (see http://sti.bmj.com/supplemental). Costs estimated in previous time periods were inflated to 2004 pounds sterling $(£)$ using the Hospital and Community Health Services Pay and Prices Index. ${ }^{14}$ All costs and effects were discounted at an annual rate of $3.5 \%$ in the base case. Sensitivity analyses were done using no discount rate for costs and effects, and $6 \%$ for costs and effects, and 3.5\% for costs and no discounting of effects.

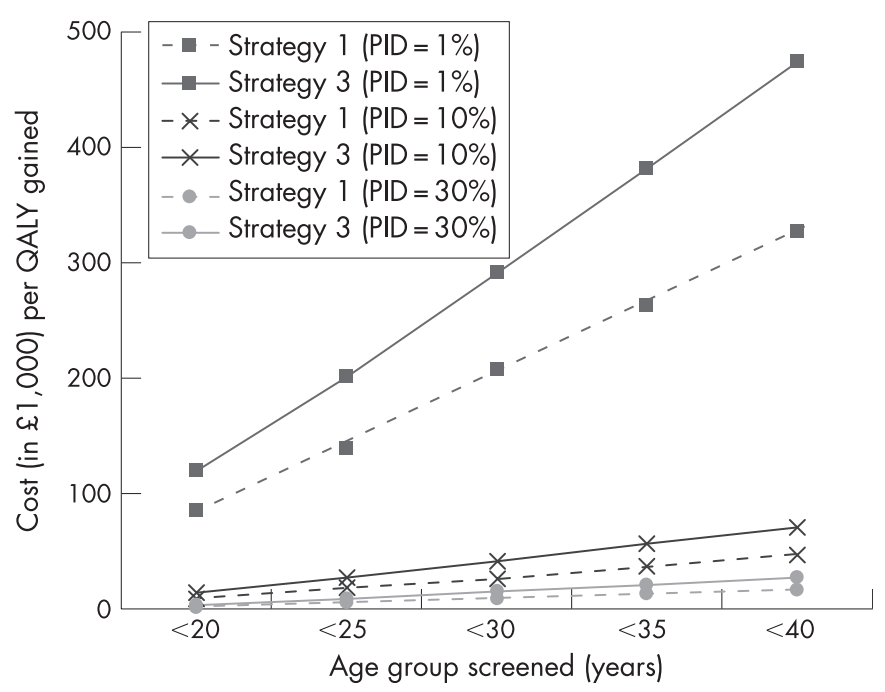

Figure 2 The average cost effectiveness of screening strategies 1 (offer women annual screen) and 3 (offer women and men annual screen) in different age groups compared to no screening, under different assumptions about pelvic inflammatory disease (PID) progression.
Two outcomes were considered in the analysis: the number of major outcomes averted (MOA) and quality adjusted life year (QALY) gained. The MOAs included cases of epididymitis, PID, EP, TFI, and neonatal conjunctivitis and neonatal pneumonia. Details of the QALY estimates for each condition are given in the appendix (on the STI website, see http:// sti.bmj.com/supplemental).

The average cost effectiveness ratio (CER) was used to compare each strategy with no screening. The CER was calculated as: (difference in costs)/(difference in benefits), between screening and no screening, where the benefits are either MOAs or QALYs gained. However, as recommended by NICE, an incremental cost effectiveness ratio (ICER) analysis was also done to assess the relative cost effectiveness of alternative screening strategies. ${ }^{17}$ The ICER was calculated by ranking the programmes in order of net costs, and the additional benefits and additional costs of each programme compared with the previous strategy (excluding dominated ones) were estimated. Programmes were dominated if they cost more than the previous strategy and resulted in fewer benefits. Both the CER and ICER were estimated separately for each assumption about the progression to PID.

The time horizon for analysing the effects of screening was 10 years. Chronic complications in women (EP, TFI) and the associated costs that occurred until a woman was 44 years old were also included.

A probabilistic multivariate sensitivity analysis was performed to assess the uncertainty of model assumptions using @ Risk (version 4.5, Palisade Corporation) running within Excel (version 2000, Microsoft). For each dynamic model simulation result (100 total for each screening strategy), the economic model was run 100 times, and for each realisation a different value for input parameters was randomly sampled from their distributions (through Latin hypercube sampling). Details of the distributions are given in the tables and the appendix (see http://sti.bmj.com/supplemental). For the multivariate sensitivity analysis, PID progression was assumed to be $10 \%$. The ICER was estimated for the costs and effects of no screening and the top four screening strategies.

\section{RESULTS \\ PID progression}

The assumption about the probability of progression to PID had a large impact on the results. The average annual incidence of predicted by the model was $58(\mathrm{PID}=1 \%)$, $581(\mathrm{PID}=10 \%)$, and $1750(\mathrm{PID}=30 \%)$ per 100000 women for a PID progression of $1 \%, 10 \%$, and $30 \%$, respectively. A study PID found 30\% (42/ 140) of PID cases had evidence of ever being exposed to chlamydial infection. ${ }^{25}$ If that is applied to the numbers seen in GP surgeries, then an estimated maximum of between 450 and 720 cases of PID per 100000 annually seen in GP surgeries may be caused by chlamydia. This suggests an estimate of around $10 \%$ progression to PID is the most likely of the PID scenarios modelled.

\section{Cost effectiveness}

Under the baseline scenario without screening, in a model population of 40000 sexually active individuals, there were on average 1392 major outcomes and 65 QALYs lost over 10 years (assuming a PID progression probability of 10\%). For different PID progression probabilities there were on average $393(1 \%$ PID) and 3529 (30\% PID) MOs, corresponding to 10 and 156 QALYs lost, respectively.

The average cost effectiveness of different screening strategies (screening versus no screening) is presented in figure 2 and in tables 3-5 (results are ranked according to increasing costs). Strategy 1 was the least effective strategy, but most cost 
Table 3 Cumulative major outcomes, quality adjusted life years lost, and costs expected over 10 years, the incremental cost per outcome for each screening strategy, and the average cost per outcome (compared to no screening) for each assumption about pelvic inflammatory disease (PID) progression: $\mathrm{PID}=1 \%$

\begin{tabular}{|c|c|c|c|c|c|c|c|}
\hline & $\begin{array}{l}\text { Total } \\
\text { MO }\end{array}$ & $\begin{array}{l}\text { Total } \\
\text { QALYs lost }\end{array}$ & Total cost (£) & $\begin{array}{l}\text { Incremental cost } \\
\text { (£)/MOA }\end{array}$ & $\begin{array}{l}\text { Incremental cost } \\
\text { (£)/QALY gained }\end{array}$ & $\begin{array}{l}\text { Average cost } \\
\text { (£)/MOA }\end{array}$ & $\begin{array}{l}\text { Average cost } \\
\text { (£)/QALY gained }\end{array}$ \\
\hline $\begin{array}{l}\text { Baseline, no } \\
\text { screening }\end{array}$ & 393 & 10 & 108408 & - & - & - & - \\
\hline Strategy $1<20$ & 256 & 6 & 430991 & 2364 & 84337 & 2364 & 84337 \\
\hline Strategy $2<20$ & 222 & 5 & 670680 & 7118 & 241271 & 3305 & 116693 \\
\hline Strategy $3<20$ & 201 & 5 & 739267 & 3125 & 149,745 & 3284 & 119562 \\
\hline Strategy $1<25$ & 215 & 5 & 811689 & Dominated & Dominated & 3960 & 139219 \\
\hline Strategy $1<30$ & 203 & 5 & 1196464 & Dominated & Dominated & 5754 & 207198 \\
\hline Strategy $2<25$ & 171 & 4 & 1378328 & 21573 & 736387 & 5728 & 206685 \\
\hline Strategy $3<25$ & 137 & 3 & 1494862 & 3474 & 157304 & 5432 & 201371 \\
\hline Strategy $1<35$ & 189 & 4 & 1577516 & Dominated & Dominated & 7204 & 262845 \\
\hline Strategy $1<40$ & 185 & 4 & 1959279 & Dominated & Dominated & 8905 & 326900 \\
\hline Strategy $2<30$ & 149 & 3 & 2088871 & Dominated & Dominated & 8122 & 296053 \\
\hline Strategy $3<30$ & 114 & 3 & 2253126 & 32374 & 1544567 & 7696 & 290770 \\
\hline Strategy $2<35$ & 140 & 3 & 2799862 & Dominated & Dominated & 10657 & 389895 \\
\hline Strategy $3<35$ & 104 & 2 & 3015808 & 75208 & 3161809 & 10067 & 381688 \\
\hline Strategy $2<40$ & 133 & 3 & 3517839 & Dominated & Dominated & 13157 & 485712 \\
\hline Strategy $3<40$ & 94 & 2 & 3773363 & 76841 & 6909379 & 12271 & 474314 \\
\hline
\end{tabular}

MO, major outcome; MOA, major outcomes averted. Values in the table are rounded for presentation. QALY, quality adjusted life years; All costs and effects are discounted at $3.5 \%$. Results are presented in rank order of total costs, which include costs of infection, complications, and programme costs. Dominated means that the MOA or QALYs gained is less than the non-dominated strategy above it in the table.

effective (that is, lowest average cost per MOA or QALY gained). Strategies 2 and 3 yielded similar results and were less cost effective than strategy 1 . Extending a strategy to include older ages resulted in smaller increases in health than costs, thereby increasing the CER. The average CER of the NCSP strategy under baseline assumptions and 10\% PID progression was $£ 27269$. None of the screening programmes modelled were cost saving.

Results of the incremental cost effectiveness analyses comparing alternative strategies are given in tables 3-5. A high ICER corresponds to a small increase in benefit over the screening programme above it but with a relatively large additional cost. The rank order of screening scenarios was the same in the incremental analysis for all assumptions about PID progression. If PID progression were $1 \%$, the ICER was very high (over $£ 80000$ per QALY gained) for any screening programme compared to no screening. For PID progression of
$10 \%$ or higher, the incremental cost per QALY gained when strategies 1, 2, and 3 (aged under 20 years) were added was below $£ 20000-£ 30000$ per QALY gained. However, adding screening of older age groups resulted in high ICERs (over $£ 50000)$.

\section{Sensitivity analyses}

The sensitivity of the estimated cost effectiveness to the intervention assumptions given the NCSP strategy (strategy 3, $<25$ years) is presented in table 6 . Low acceptance resulted in a higher CER compared to the baseline of 50\% acceptance. Increasing the effective partner notification rate from $20 \%$ to $50 \%$ reduced the cost effectiveness ratio by about $10 \%$. Offering men and women aged under 25 years a single screening test was more cost effective than continuous screening, mainly because of the much lower costs. The impact of changing the discount rate is given in table 7 .

Table 4 Cumulative major outcomes, quality adjusted life years lost, and costs expected over 10 years, the incremental cost per outcome for each screening strategy, and the average cost per outcome (compared to no screening) for each assumption about pelvic inflammatory disease (PID) progression: PID $=10 \%$

\begin{tabular}{|c|c|c|c|c|c|c|c|}
\hline & $\begin{array}{l}\text { Total } \\
\text { MO }\end{array}$ & $\begin{array}{l}\text { Total } \\
\text { QALYs lost }\end{array}$ & Total cost $(£)$ & $\begin{array}{l}\text { Incremental cost } \\
\text { (£)/MOA }\end{array}$ & $\begin{array}{l}\text { Incremental cost } \\
\text { (£)/QALY gained }\end{array}$ & $\begin{array}{l}\text { Average cost } \\
\text { (£)/MOA }\end{array}$ & $\begin{array}{l}\text { Average cost } \\
\text { (£)/QALY gained }\end{array}$ \\
\hline $\begin{array}{l}\text { Baseline, no } \\
\text { screening }\end{array}$ & 1392 & 65 & 310695 & - & - & - & - \\
\hline Strategy $1<20$ & 883 & 39 & 553352 & 477 & 9204 & 477 & 9204 \\
\hline Strategy $2<20$ & 736 & 31 & 771367 & 1484 & 29416 & 703 & 13640 \\
\hline Strategy $3<20$ & 673 & 29 & 832498 & 959 & 24103 & 726 & 14371 \\
\hline Strategy $1<25$ & 739 & 32 & 918213 & Dominated & Dominated & 930 & 18476 \\
\hline Strategy $1<30$ & 645 & 28 & 1283628 & 16415 & 978039 & 1303 & 26459 \\
\hline Strategy $2<25$ & 584 & 24 & 1462,494 & 2928 & 44109 & 1426 & 28212 \\
\hline Strategy $3<25$ & 468 & 19 & 1556572 & 807 & 19352 & 1348 & 27269 \\
\hline Strategy $1<35$ & 633 & 28 & 1666599 & Dominated & Dominated & 1788 & 36849 \\
\hline Strategy $1<40$ & 610 & 28 & 2048769 & Dominated & Dominated & 2224 & 46404 \\
\hline Strategy $2<30$ & 491 & 20 & 2157585 & Dominated & Dominated & 2051 & 41470 \\
\hline Strategy $3<30$ & 400 & 17 & 2308023 & 11059 & 302328 & 2013 & 41461 \\
\hline Strategy $2<35$ & 460 & 20 & 2869275 & Dominated & Dominated & 2745 & 56481 \\
\hline Strategy $3<35$ & 363 & 16 & 3064432 & 20479 & 747964 & 2676 & 55987 \\
\hline Strategy $2<40$ & 444 & 20 & 3582115 & Dominated & Dominated & 3453 & 71953 \\
\hline Strategy $3<40$ & 343 & 15 & 3828432 & 39230 & 1938410 & 3355 & 70952 \\
\hline
\end{tabular}

MO, major outcome; MOA, major outcomes averted. Values in the table are rounded for presentation. QALY, quality adjusted life years; All costs and effects are discounted at $3.5 \%$. Results are presented in rank order of total costs, which include costs of infection, complications, and programme costs. Dominated means that the MOA or QALYs gained is less than the non-dominated strategy above it in the table. 
Table 5 Cumulative major outcomes, quality adjusted life years lost, and costs expected over 10 years, the incremental cost per outcome for each screening strategy, and the average cost per outcome (compared to no screening) for each assumption about pelvic inflammatory disease (PID) progression: $\mathrm{PID}=30 \%$

\begin{tabular}{|c|c|c|c|c|c|c|c|}
\hline & Total MO & $\begin{array}{l}\text { Total } \\
\text { QALYs lost }\end{array}$ & $\begin{array}{l}\text { Total cost } \\
\text { (£) }\end{array}$ & $\begin{array}{l}\text { Incremental cost } \\
\text { (£)/MOA }\end{array}$ & $\begin{array}{l}\text { Incremental cost } \\
\text { (£)/QALY gained }\end{array}$ & $\begin{array}{l}\text { Average cost } \\
\text { (£)/MOA }\end{array}$ & $\begin{array}{l}\text { Average cost } \\
\text { (£)/QALY gained }\end{array}$ \\
\hline $\begin{array}{l}\text { Baseline, no } \\
\text { screening }\end{array}$ & 3529 & 156 & 709068 & - & - & - & - \\
\hline Strategy $1<20$ & 2216 & 92 & 796042 & 66 & 1364 & 66 & 1364 \\
\hline Strategy $2<20$ & 1878 & 75 & 974854 & 529 & 10402 & 161 & 3283 \\
\hline Strategy $3<20$ & 1676 & 66 & 1008678 & 168 & 3845 & 162 & 3338 \\
\hline Strategy $1<25$ & 1799 & 75 & 1110924 & Dominated & Dominated & 232 & 4960 \\
\hline Strategy $1<30$ & 1641 & 70 & 1466413 & 13279 & Dominated & 401 & 8799 \\
\hline Strategy $2<25$ & 1397 & 55 & 1600015 & 546 & 53317 & 418 & 8834 \\
\hline Strategy $3<25$ & 1195 & 46 & 1682280 & 407 & 8961 & 417 & 8845 \\
\hline Strategy $1<35$ & 1574 & 68 & 1842956 & Dominated & Dominated & 580 & 12987 \\
\hline Strategy $1<40$ & 1508 & 66 & 2213265 & Dominated & Dominated & 744 & 16829 \\
\hline Strategy $2<30$ & 1200 & 48 & 2277375 & Dominated & Dominated & 673 & 14589 \\
\hline Strategy $3<30$ & 1018 & 41 & 2419181 & 4181 & 149930 & 681 & 14877 \\
\hline Strategy $2<35$ & 1138 & 47 & 2991631 & Dominated & Dominated & 955 & 21068 \\
\hline Strategy $3<35$ & 909 & 38 & 3163011 & 6835 & 238076 & 937 & 20783 \\
\hline Strategy $2<40$ & 1071 & 46 & 3696199 & Dominated & Dominated & 1215 & 27228 \\
\hline Strategy $3<40$ & 852 & 37 & 3921645 & 13304 & 714049 & 1200 & 26966 \\
\hline
\end{tabular}

MO, major outcome; MOA, major outcomes averted. Values in the table are rounded for presentation. QALY, quality adjusted life years; All costs and effects are discounted at $3.5 \%$. Results are presented in rank order of total costs, which include costs of infection, complications, and programme costs. Dominated means that the MOA or QALYs gained is less than the non-dominated strategy above it in the table.

\section{Uncertainty analysis}

Figure 3 illustrates the range of likely results from the probabilistic sensitivity analysis on the ICER (PID progression $=10 \%$ ). There is considerable uncertainty, even in the no screening scenario, particularly in the QALYs lost from chlamydia (the spread in the horizontal axis is greater than in the vertical). It is clear from figure 3 that strategy $1 \quad(<20$ years) results in large incremental QALY gains and has a high probability of falling below $£ 20000$ per QALY gained (at 10\% PID progression). Moving to strategy 2 ( $<20$ years) results in almost half the points lying above the $£ 30000$ per QALY gained line. Including men (strategy $3,<20$ years) results in small additions to the cost of the programme and small additional benefits over strategy 2, and about half of the simulations fall below $£ 20000$ per QALY gained. Increasing the programme further (strategy $1,<30$ years), would result in large additional costs and few additional benefits, with nearly all results falling above $£ 30000$ per QALY gained.

\section{DISCUSSION}

Estimates of the costs and cost effectiveness of different chlamydia screening strategies including the current strategy recommended by the NCSP (strategy 3, annual screening offer to women and men aged under 25 years) are presented. None of the screening strategies modelled were cost saving, but all resulted in better health and fewer major outcomes.

The most influential parameter was the probability of cases progressing to PID. Most other cost effectiveness studies of chlamydia screening have used an estimate of around $25-30 \%$ progression to PID (including both symptomatic and asymptomatic PID).$^{26}$ However, a recent study by van Valkengoed et al based on Dutch data concluded that the risk of PID after a

Table 6 Sensitivity of the estimated average cost effectiveness of screening to the choice of intervention parameter

\begin{tabular}{|c|c|c|c|c|c|c|}
\hline PID rate & Scenario & Net MOA & Net QALY & $\begin{array}{l}\text { Net costs } \\
\text { (£) }\end{array}$ & $\begin{array}{l}\text { Cost } \\
\text { (£)/MOA }\end{array}$ & $\begin{array}{l}\text { Cost } \\
\text { (£)/QALY gained }\end{array}$ \\
\hline \multirow[t]{7}{*}{$1 \%$} & Screening baseline & 255 & 7 & 1386454 & 5432 & 201371 \\
\hline & Acceptance $=F, 10 \% ; M, 1.4 \%$ & 70 & 2 & 1290587 & 18308 & 643037 \\
\hline & Acceptance $=10 \%$ & 117 & 3 & 1315002 & 11240 & 407440 \\
\hline & Acceptance $=30 \%$ & 220 & 6 & 1356937 & 6182 & 231433 \\
\hline & Acceptance $=70 \%$ & 275 & 7 & 1404474 & 5101 & 190166 \\
\hline & $\mathrm{PN}=50 \%$ & 286 & 8 & 1415138 & 4953 & 186321 \\
\hline & Screen only once & 187 & 5 & 530449 & 2830 & 104007 \\
\hline \multirow[t]{7}{*}{$10 \%$} & Screening baseline & 924 & 46 & 1245877 & 1348 & 27269 \\
\hline & Acceptance $=F, 10 \% ; M, 1.4 \%$ & 302 & 15 & 1241250 & 4106 & 83717 \\
\hline & Acceptance $=10 \%$ & 443 & 22 & 1245655 & 2809 & 57445 \\
\hline & Acceptance $=30 \%$ & 807 & 40 & 1234664 & 1530 & 30869 \\
\hline & Acceptance $=70 \%$ & 989 & 49 & 1256063 & 1270 & 25633 \\
\hline & $\mathrm{PN}=50 \%$ & 1021 & 50 & 1257727 & 1232 & 24966 \\
\hline & Screen only once & 677 & 34 & 429762 & 635 & 12814 \\
\hline \multirow[t]{7}{*}{$30 \%$} & Screening baseline & 2334 & 110 & 973212 & 417 & 8845 \\
\hline & Acceptance $=F, 10 \% ; M, 1.4 \%$ & 762 & 35 & 1156289 & 1518 & 33241 \\
\hline & Acceptance $=10 \%$ & 1121 & 51 & 1115870 & 995 & 21676 \\
\hline & Acceptance $=30 \%$ & 2030 & 95 & 1005087 & 495 & 10605 \\
\hline & Acceptance $=70 \%$ & 2481 & 117 & 969306 & 391 & 8320 \\
\hline & $\mathrm{PN}=50 \%$ & 2599 & 122 & 960098 & 369 & 7899 \\
\hline & Screen only once & 1735 & 81 & 227799 & 131 & 2826 \\
\hline
\end{tabular}

NSCP, National Chlamydia Screening Programme; PID, pelvic inflammatory disease; MOA, major outcomes averted; QALY, quality adjusted life years; PN, partner notification, $F$, female; $M$, male. Under baseline assumptions, screening acceptance is $50 \%$, PN is $20 \%$, and screening is offered annually.

The baseline is the NSCP strategy (strategy 3, annual screen offer to men and women under 25 years old) compared to no screening. 
Table 7 Sensitivity of the results to the discount rate for costs and effects, NCSP strategy (strategy 3, annual screening offer to men and women aged under 25 years compared with no screening).

\begin{tabular}{|c|c|c|c|c|c|c|c|}
\hline $\begin{array}{l}\text { PID } \\
\text { rate }\end{array}$ & $\begin{array}{l}\text { Discount } \\
\text { rate effects } \\
\text { (\%) }\end{array}$ & $\begin{array}{l}\text { Discount } \\
\text { rate costs } \\
(\%)\end{array}$ & Net MOA & Net QALY & Net costs $(£)$ & $\begin{array}{l}\text { Cost } \\
\text { (£)/MOA }\end{array}$ & $\begin{array}{l}\text { Cost } \\
\text { (£)/QALY gained }\end{array}$ \\
\hline \multirow[t]{4}{*}{$1 \%$} & 0 & 0 & 321 & 11 & 1644897 & 5118 & 144924 \\
\hline & 3.5 & 3.5 & 255 & 7 & 1386454 & 5432 & 201371 \\
\hline & 0.0 & 3.5 & 321 & 11 & 1383644 & 4305 & 121907 \\
\hline & 6 & 6 & 219 & 5 & 1236641 & 5641 & 243833 \\
\hline \multirow[t]{4}{*}{$10 \%$} & 0 & 0 & 1187 & 81 & 1406086 & 1185 & 17265 \\
\hline & 3.5 & 3.5 & 924 & 46 & 1245877 & 1348 & 27269 \\
\hline & 0.0 & 3.5 & 1187 & 81 & 1220846 & 1029 & 14991 \\
\hline & 6 & 6 & 786 & 32 & 1131554 & 1439 & 35620 \\
\hline \multirow[t]{4}{*}{$30 \%$} & 0 & 0 & 2996 & 197 & 959671 & 320 & 4872 \\
\hline & 3.5 & 3.5 & 2334 & 110 & 973212 & 417 & 8845 \\
\hline & 0.0 & 3.5 & 2996 & 197 & 911004 & 304 & 4625 \\
\hline & 6 & 6 & 1987 & 76 & 922869 & 464 & 12081 \\
\hline
\end{tabular}

NSCP, National Chlamydia Screening Programme; PID, pelvic inflammatory disease; MOA, major outcomes averted; QALY, quality adjusted life years.

chlamydial infection is likely to be less than $1 \% .^{12}$ Another study by Morré et al followed up 30 asymptomatically infected women and none developed PID after 1 year of follow up. ${ }^{11}$ If $30 \%$ of women with asymptomatic chlamydial infection progress to PID, we would expect a much higher reported incidence of PID in general practice than is observed. Although some cases may be undiagnosed, the number of reported cases of PID in general practice is likely to be a reasonable upper bound on the number of cases caused by chlamydial infection, since this is PID from all causes including misdiagnosis. ${ }^{13}$ In fact the number of reported cases is inconsistent with progression greater than about $10 \%$. This has major implications for the results of the cost effectiveness analysis (tables 35).

If we were to consider solely the NCSP strategy compared to no screening, the average cost effectiveness ratio is about $£ 27000$ when PID progression is 10\%. NICE suggest that programmes with an ICER of greater than $£ 20000-£ 30000$ per QALY gained are unlikely to be accepted on cost effectiveness grounds. ${ }^{17}$ Therefore, the NCSP strategy appears to be acceptable on cost effectiveness grounds if we ignore other screening strategies. However, NICE recommends that the incremental cost effectiveness ratio of alternative strategies is also explored. ${ }^{17}$ This indicates that the NCSP strategy involves a relatively high expected cost compared to the additional expected benefits. If PID progression were $10 \%$ or higher, then the full incremental analysis suggests that screening men and women aged under 20 years should be recommended. If only $1 \%$ of infected women develop PID, then none of the screening strategies appeared to be acceptable on cost effectiveness grounds.

The sensitivity analyses highlighted how the current strategy could be made more cost effective. Increased acceptance rates result in more favourable cost effectiveness results compared to baseline (table 6). The high CER for low acceptance occurs from the costs not only for those who accept screening but who also do not accept a screen, ${ }^{27}$ in addition to the costs of complications. Efforts could be made to raise awareness about chlamydia and the benefits of regularly obtaining a screen to improve acceptance rates. Additionally, results from the third year of the NCSP indicate that $33 \%$ of partners were treated, ${ }^{3}$ which is higher than our baseline assumption of $20 \%$ and would make screening more cost effective. Finally, the model used in this analysis was fitted to data from a review of chlamydia prevalence studies in women, but no equivalent data were available on male prevalence. ${ }^{1} \mathrm{New}$ evidence from the NCSP and surveillance from STI clinics suggest that the peak prevalence is in men aged $20-25 .^{32}$ Future analyses could
- A. Baseline: no screening

- B. Strategy $1(<20$ year) vs no screening

- C. Strategy $2(<20$ year) vs strategy $1(<20$ year)

- D. Strategy $3(<20$ year) vs strategy $2(<20$ year)

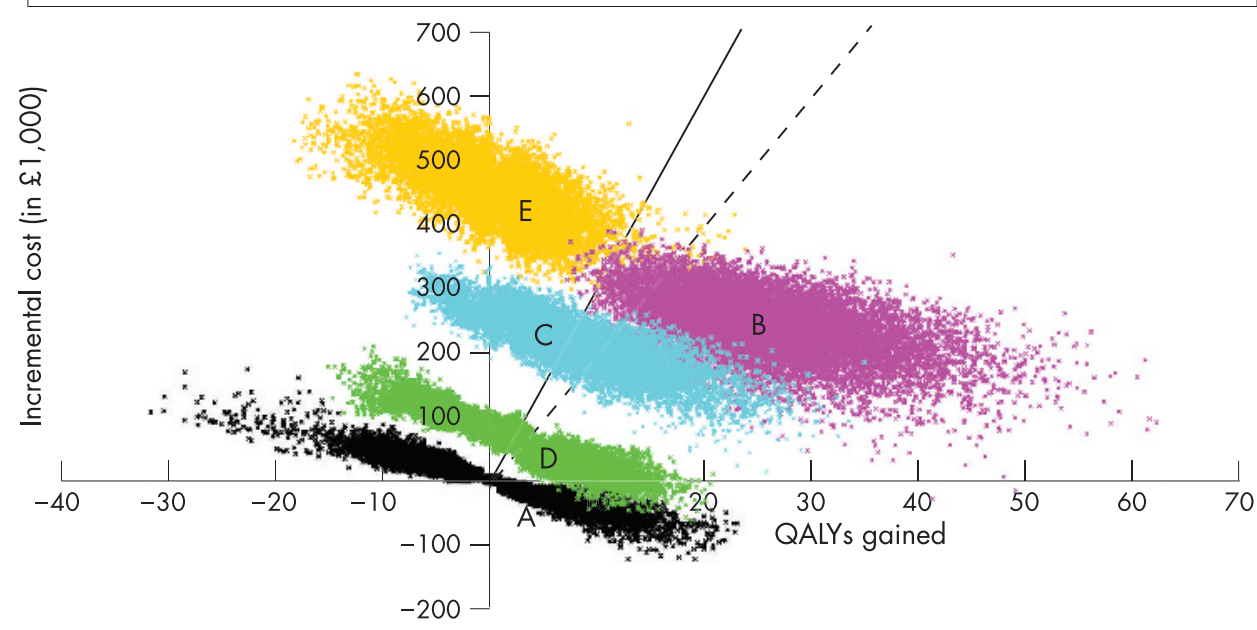

Figure 3 Multivariate sensitivity analysis of the estimated incremental costs and quality adjusted life year (QALY) gain for the most cost effective screening programmes, assuming pelvic inflammatory disease progression of $10 \%$. 
include new data to reflect these changes, which may in turn impact on the results.

A few papers have estimated the cost effectiveness of chlamydia screening using dynamic models ${ }^{20} 20$; however, most studies have used static models, which are incapable of including population level effects. ${ }^{26}{ }^{31}$ Welte et al ${ }^{20}$ used an appropriate dynamic model similar to ours to examine the cost effectiveness of screening in the Netherlands. They estimated that screening might be cost saving after 10 years. The disparity in these results from ours is likely to be due to three key differences in their assumptions in both their dynamic and cost effectiveness models. Firstly, they assumed a high proportion of individuals being treated as symptomatic cases before screening introduction $\left(\sim 40 \%\right.$ compared with under $5 \%$ in our $\left.\operatorname{model}^{4}\right)$, thereby effectively removing them from developing complications. Secondly, they assumed a high probability of PID progression $(25 \%)$. Thirdly, costs for most complications were much higher than those assumed in our model. For example, they assumed that $25 \%$ of PID cases will be admitted to hospital inpatient care, including an 11 day hospital stay, yielding an average estimated cost that was over six times higher than ours. The costs of other complications (EP, TFI, neonatal complications, epididymitis) were also higher than our estimates.

The screening costs in the current analysis were taken from a chlamydia screening pilot study. ${ }^{27}{ }^{32}$ The initial set-up costs of a national chlamydia screening programme are likely to include costs not modelled in this analysis, including training costs, computerisation costs, personnel, etc. Therefore this analysis may underestimate the true costs of a screening programme, thereby making screening appear more favourable than it may be. Additionally, in accordance with the NICE guidelines, in this study only the direct medical and screening costs were examined. Another large population based chlamydia screening study is being conducted which includes an analysis of patient costs. ${ }^{33}$ These could be included in further analyses, along with other societal costs. Finally, costs associated with false positive or false negative tests were not considered in this analysis. False positive tests result in costs due to treatment and partner follow-up. If chlamydia prevalence declines, the probability of false positive results increases. Individuals with false positive tests may incur psychological and social costs associated with disclosure of diagnosis to sex partners and stigma attached to STIs, with no compensating benefit resulting from treatment gained by those infected. ${ }^{34-37}$ Therefore, there may be QALY loss from screening itself, which should be further investigated.

In this analysis two outcomes were used: MOAs and QALYs gained. MOAs are an intermediate outcome, and it is difficult to compare results with other health interventions. However, only one other cost effectiveness study has used QALYs for chlamydia screening. ${ }^{26}{ }^{38} \mathrm{Hu}$ et al also used the Institute of Medicine values, ${ }^{21}{ }^{38}$ as these are the only estimates currently available. The QALY estimates could be improved in future studies to better understand the health loss from chlamydial infection, complications, and screening.

This study used a dynamic model to estimate the likely cost effectiveness of chlamydia screening strategies. Results can be used to inform decisions about which screening strategies may be the most beneficial in the context of limited healthcare resources. It suggests that offering an annual screen to men and women under 25 years of age result in ICERs above the normally accepted levels when compared with screening only those aged under 20 years (although this strategy may be deemed cost effective when compared with "no screening"). Results suggest that increasing screening acceptance and effective partner notification may yield a more favourable cost effectiveness ratio owing to greater benefits without a large relative increase in costs. Since one of the greatest uncertainties
Key messages

- All chlamydia screening strategies modelled are likely to improve health and cost money.

- Screening men and women aged under 25 years (NCSP strategy) appears to be cost effective compared to no screening; results suggest that a less inclusive strategy may be more acceptable on cost effectiveness grounds.

- The results depend largely on the assumed progression from chlamydial infection to PID, which is likely to be lower than that used previously in cost effectiveness studies.

- A realistic model of infection, disease progression, and costs can be used to estimate the likely cost effectiveness of a national chlamydia screening programme which in turn can be used to advise public health policy decisions.

that impacts on the results is the probability of progression to PID, future work should focus on understanding its natural history. Monitoring the incidence of PID as screening is introduced nationally should be a research priority.

\section{ACKNOWLEDGEMENTS}

We would like to thank Iona Martin for advice about laboratory testing, Alessia Melegaro for HES extraction and help analysing the data, members of the STI Unit and Modelling \& Economic Unit of the HPA, and members of the NCSP team including Lynsey Emmett, Teresa Battison, and Ian Simms for comments on the paper.

Contributors: EJA was the primary author, constructed the cost effectiveness model and analysed and interpreted the results. KMET coded changes to the dynamic simulation model, helped interpret the results, and revised the manuscript. WJE assisted with model development, interpretation of results and revised the manuscript.

\section{Authors' affiliations}

Elisabeth J Adams, W John Edmunds, Modelling \& Economics Unit, Health Protection Agency, 61 Colindale Avenue, Colindale, London NW9 5EQ, UK

Katherine M E Turner, Infectious Disease Epidemiology, Imperial College, St Mary's Campus, Norfolk Place, London W2 IPG, UK

Funding: Funding for the work was provided by the Health Protection Agency and the Department of Health (England).

Conflict of interest: none.

\section{REFERENCES}

1 Adams EJ, Charlett A, Edmunds WJ, et al. Chlamydia trachomatis in the United Kingdom: a systematic review and analysis of prevalence studies. Sex Transm Infect 2004;80:354-62.

2 LaMontagne DS, Fenton KA, Randall S, et al. Establishing the National Chlamydia Screening Programme in England: results from the first full year of screening. Sex Transm Infect 2004;80:335-41.

3 National Chlamydia Screening Steering Group. New frontiers: annual report of the National Chlamydia Screening Programme in England 2005/06. London: Health Protection Agency, 2006.

4 Turner KME Adams EJ, Gay NJ, et al. Developing a realistic sexual network model of chlamydia transmission in Britain. Theor Biol Med Model 2006;3:3.

5 Turner KME, Adams EJ, LaMontagne DS, et al. Modelling the effectiveness of chlamydia screening in England. Sex Transm Infect 2006;82:496-502.

6 LaMontagne DS, Baster K, Emmett L, et al. Incidence and re-infection rates of genital chlamydial infection among women aged 16-24 years attending general practice, family planning and genitourinary medicine clinics in England: a prospective cohort study. Sex Transm Infect 2007;83:292-303.

7 National Chlamydia Screening Steering Group. Looking back, moving forward. Annual report of the National Chlamydia Screening Programme in England, 2004-5. London: Department of Health, 2005.

8 Hermann B. The fall and rise of chlamydia in Sweden: the role of opportunistic screening. Amsterdam, The Netherlands: 16th Biennial meeting of the International Society for Sexually Transmitted Diseases Research (ISSTDR), 2005. 
9 Weström L, Joesoef R, Reynolds G, et al. Pelvic inflammatory disease and fertility: a cohort study of 1,844 women with laparoscopically verified disease and 657 control women with normal laparoscopic results. Sex Transm Dis 1992;19:185-92.

10 Stamm W, Guinan M, Johnson C, et al. Effect of treatment regimens for Neisseria gonorrhoeae on simultaneous infection with Chlamydia trachomatis. NEngl J Med 1984;310:545-9.

11 Morré S, van den Brule A, Rozendaal L, et al. The natural course of asymptomatic Chlamydia trachomatis infections: $45 \%$ clearance and no development of clinical PID after one-year follow-up. Int J STD AIDS 2002;13(Suppl 2):12-8.

12 Van Valkengoed IG, Morre SA, van Den Brule AJ, et al. Overestimation of complication rates in evaluations of Chlamydia trachomatis screening programmes--implications for cost-effectiveness analyses. Int J Epidemiol 2004;33:416-25.

13 Hughes G, Williams T, Simms I, et al. Trends in diagnoses of genital chlamydia and pelvic inflammatory disease in primary care, 1990-2003: analysis of data in the General Practice Research Database. Annual Conference; 2004 Sep 14; University of Warwick: Health Protection Agency, 2004.

14 Curtis L, Netten A. Unit costs of health and social care 2004. Kent: Personal Social Services Research Unit, University of Kent, 2004.

15 Joint Formulary Committee. British National Formulary No 47. London: British Medical Association and Royal Pharmaceutical Society of Great Britain, 2004

16 Department of Health. Reference costs 2004, www.dh.gov.uk, 2005.

17 National Institute for Health and Clinical Excellence. Guide to the methods of technology appraisal. London: NICE, 2004.

18 Rosenman MB, Mahon BE, Downs SM, et al. Oral erythromycin prophylaxis vs watchful waiting in caring for newborns exposed to Chlamydia trachomatis. Arch Pediatr Adolesc Med 2003;157:565-71

19 Welte R, Jager J, Postma M. Cost-effectiveness of screening for genital Chlamydia trachomatis. Exp Rev Pharmacoeconom Outcomes Res 2001;1:145-56.

20 Welte R, Kretzschmar M, Leidl R, et al. Cost-effectiveness of screening programs for Chlamydia trachomatis: a population-based approach. Sex Transm Dis 2000;27:518-29.

21 Institute of Medicine. Vaccines for the 21 st century: a tool for decision making. Washington, DC: National Academy Press, 2000.

22 Department of Health. Hospital Episode Statistics. www. hesonline.org.uk, 2004.

23 Thurmond AS, Rosch J. Nonsurgical fallopian tube recanalization for treatment of infertility. Radiology 1990;174:371-4.

24 Collins JA, Feeny D, Gunby J. The cost of infertility diagnosis and treatment in Canada in 1995. Hum Reprod 1997; 12:951-8.

25 Simms I, Stephenson JM, Mallinson H, et al. Risk factors associated with pelvic inflammatory disease. Sex Transm Infect 2006;82:452-7.

26 Roberts TE, Robinson S, Barton P, et al. Screening for Chlamydia trachomatis: a systematic review of the economic evaluations and modelling. Sex Transm Infect 2006;82:193-200.

27 Adams EJ, LaMontagne DS, Johnston AR, et al. Modelling the healthcare costs of an opportunistic chlamydia screening programme. Sex Transm Infect 2004;80:363-70.

28 Health Protection Agency. A complex picture: HIV and other sexually transmitted infections in the United Kingdom: 2006. London: Health Protection Agency, Centre for Infections, 2006.

29 Townshend JRP, Turner HS. Analysing the effectiveness of chlamydia screening. J Oper Res Soc 2000;51:812-24.

30 De Vries R, van Bergen JE, de Jong-van den Berg LT, et al. Systematic screening for Chlamydia trachomatis: estimating cost-effectiveness using dynamic modeling and Dutch data. Value Health 2006;9:1-11

31 Welte R, Postma $M$, Leidl $R$, et al. Costs and effects of chlamydial screening dynamic versus static modeling. Sex Transm Dis 2005;32:474-83.

32 Pimenta JM, Catchpole M, Rogers PA, et al. Opportunistic screening for genital chlamydial infection. II: Prevalence among healthcare attenders, outcome, and evaluation of positive cases, Sex Transm Infect 2003;79:22-7.

33 Robinson SM, Roberts TE, Barton PM, et al. The health care and patient costs of an proactive chlamydia screening programme: the Chlamydia Screening Studies (ClaSS) project. Sex Transm Infect. 2007, doi: 10, 1136/sti.2006.023374.

34 Pavlin NL, Gunn JM, Parker R, et al. Implementing chlamydia screening: what do women think? A systematic review of the literature. BMC Public Health 2006:6:221.

35 Duncan B, Hart G, Scoular A, et al. Qualitative analysis of psychosocial impact of diagnosis of Chlamydia trachomatis: implications for screening. BMJ 2001;322:195-9.

36 Mills N, Daker-White G, Graham A, et al. Population screening for Chlamydia trachomatis infection in the UK: a qualitative study of the experiences of those screened. Fam Pract 2006;23:550-7.

37 Santer M. Screening for genital chlamydial infection in women in general practice: psychological effects of such screening are important. BMJ 1997;315:1540-1.

38 Hu D, Hook EWI, Goldie SJ. Screening for Chlamydia trachomatis in women 15 to 29 years of age: a cost-effectiveness analysis. Ann Intern Med 2004; 141:501-13

39 Cates W Jr, Wasserheit JN. Genital chlamydial infections: epidemiology and reproductive sequelae. Am J Obstet Gynecol 1991;164(6 Part 2):1773-81.

40 Office for National Statistics. Birth Statistics, Series FM1 no 33. London: Stationery Office, 2005.

41 Cassell JA, Mercer CH, Sutcliffe L, et al. Trends in sexually transmitted infections in general practice 1990-2000: population based study using data from the UK general practice research database. BMJ 2006;332:332-4.

42 Walker PP, Wilson J. National guideline for the management of epididymo orchitis. London: British Association of Sexual Health \& HIV, 2001.

43 Philips Z, Barraza-Llorens M, Posnett J. Evaluation of the relative costeffectiveness of treatments for infertility in the UK. Hum Reprod 2000;15:95-106.

\section{COMMENTARY}

Adams et al cast doubt on the cost-effectiveness of opportunistic chlamydia screening, as implemented in the English National Chlamydia Screening Programme. ${ }^{1}$ A recent economic evaluation of a proactive postal screening approach has shown that this is also unlikely to be a cost-effective approach. ${ }^{2}$ These two studies, using state-of-the-art individual-based transmission dynamic network models, ${ }^{23}$ contrast sharply with almost all other published evaluations. Their results contradict not only those using an inappropriate modelling approach, ${ }^{4}$ but also more recent studies that used dynamic modelling and found screening to be cost effective ${ }^{5}$ or even cost saving. ${ }^{6}$

Adams et al's study is an important contribution to the debate about the appropriateness of chlamydia screening programmes. ${ }^{8}$ They clearly show that a chlamydia screening intervention can appear cost effective or not, depending on the assumption made about the probability of endocervical chlamydia progressing to pelvic inflammatory disease. They concluded that opportunistic screening was not cost effective if the progression rate was below $10 \%$ and that available epidemiological data were incompatible with a higher progression rate. The recent economic evaluations using individualbased dynamic modelling that found screening to be cost saving used figures of $20 \%$ to $25 \%{ }^{6}{ }^{7}$

Decision makers such as the National Institute for Health and Clinical Excellence (NICE) use the incremental costeffectiveness ratio (ICER) for decision making. In this case, the ICER refers to the additional cost per additional unit of benefit of screening compared to the alternative of no screening. Unfortunately, Adams et al misleadingly refer to the results of their main evaluation and the sensitivity analysis as average cost-effectiveness ratios (average CERs). Their definition, "(difference in costs)/(difference in benefits) between screening and no screening" is, however, the standard definition for the ICER. ${ }^{1}{ }^{9}$ The standard unit of benefit is the quality adjusted life-year (QALY) which is a single measure summarising health improvements resulting from changes in both quality and quantity of life. The only QALY estimates for chlamydia and its complications were derived from an expert panel meeting rather than primary research, ${ }^{10}$ so their validity is not known. ${ }^{4}$ Most economic evaluations of chlamydia screening have therefore estimated the costs of screening per major outcome averted, typically including pelvic inflammatory disease, ectopic pregnancy, tubal infertility and neonatal complications..$^{2-7}$ Such studies can be compared among themselves, but are less useful for decision making because there are no agreed thresholds for cost-effectiveness measured in "natural units" such as these.

Adams et al used both measures of cost-effectiveness. They estimated that the current strategy being implemented in the National Chlamydia Screening Programme in England had an ICER of $£ 27269$ per QALY gained and $£ 1348$ per major outcome averted, compared to no screening, assuming about $40 \%$ of sexually active women and men under 25 years would be screened every year, and that $10 \%$ of untreated chlamydia cases result in pelvic inflammatory disease. They suggest that such a programme could be accepted on cost-effectiveness grounds because the ICER was below $£ 30,000$ per QALY. In fact, NICE guidance states that "Above a most plausible ICER of $£ 20,000$ / QALY, judgements about the acceptability of the technology as an effective use of NHS resources are more likely to make more explicit reference to factors including: the degree of uncertainty around the calculation of the ICERs ..." (section 6.2.6.10; page 33). ${ }^{11}$ Given the considerable uncertainty about the QALY estimates used by Adams et al this ICER, in QALY terms, exceeds the acceptable threshold approved by NICE and should be subjected to 